\title{
FUNGOS MICORRÍZICOS ARBUSCULARES ASSOCIADOS ÀS CULTURAS CULTIVADAS EM SISTEMAS AGROFLORESTAIS E EM MONOCULTURAS EM PROJETOS DE ASSENTAMENTO DO SUDESTE PARAENSE
}

\author{
Helena de Souza Corrêa ${ }^{1}$; Clarissa Mendes Knoechelmann²; Andréa Hentz de Mello³; \\ Fernanda Dias Pereira ${ }^{4}$; Fernando Michelotti ${ }^{5}$; Rosana Quaresma Maneschy ${ }^{6}$ \\ ${ }^{1}$ Discente do curso de Agronomia, Faculdade de Ciências Agrárias de Marabá (FCAM), Universidade Federal do Pará \\ (UFPA), bolsista do projeto FAPESPA, helenamedleg@ hotmail.com \\ ${ }^{2}$ Professora Assistente I, FCAM, UFPA \\ ${ }^{3}$ Professora Adjunta II, FCAM, UFPA \\ ${ }^{4}$ Discente do curso de Agronomia, FCAM, UFPA, bolsista da FAPESPA \\ ${ }^{5}$ Professor Assistente IV, FCAM, UFPA \\ ${ }^{6}$ Professora Adjunta I, FCAM, UFPA
}

\begin{abstract}
RESUMO: Diante do atual cenário de degradação dos solos cultivados, o homem vem se adaptando e buscando alternativas para produzir alimento e reduzir consideravelmente os prejuízos com a utilização de um dos recursos mais importantes para sobrevivência humana. $\mathrm{O}$ uso de estratégias biológicas, entre as quais se destacam as associações micorrízicas arbusculares, no aperfeiçoamento de sistemas de manejo que racionalizem o uso dos recursos naturais da região, é fundamental para o desenvolvimento econômico contínuo, socialmente justo e ambientalmente sustentável. Para tanto, é necessário melhor entendimento de aspectos ecológicos da comunidade de fungos micorrízicos arbusculares (FMAs). Dentro desse contexto, o objetivo desse trabalho foi avaliar a simbiose micorrízica das comunidades de FMAs associados à diversas culturas de interesse de agricultores familiares quando cultivados em sistema agroflorestal (SAF) e em monocultivo, além de estimar a diversidade de comunidades de FMAs associados às raízes destas culturas. O gênero de FMAs Glomus na região amazônica apresentaram ampla adaptação à variação ambiental em ambos os sistemas, podendo assim, serem melhores estudados na produção de inoculantes para o plantio de mudas de espécies frutíferas e nativas da Amazônia.
\end{abstract}

PALAVRAS-CHAVE: Amazônia, sistemas de produção, micorrizas.

\section{HONGOS DE MICORRIZÓGENOS ARBUSCULARES ASOCIADOS CON CULTIVOS SEMBRADOS EN SISTEMAS AGROFORESTALES Y MONOCULTURAS EN PROYECTOS DE ASENTAMIENTOS DEL SURESTE PARAENSE}

RESUMEN: En el escenario actual de degradación de las tierras cultivadas, el hombre ha ido adaptando y buscar alternativas para producir alimentos y reducir considerablemente las pérdidas con el uso de uno de los recursos más importantes para la supervivencia humana. El uso de estrategias biológicas, entre las que destacan las asociaciones de micorrizas arbusculares en la mejora de los sistemas de gestión que racionalizar el uso de los recursos naturales en la región, es fundamental para el desarrollo económico sostenido, socialmente justo y ambientalmente sostenible. Por lo tanto, es necesario comprender mejor los aspectos ecológicos de la comunidad de hongos micorrizógenos arbusculares (HMA). Dentro de este contexto, el objetivo de este estudio 
fue evaluar la simbiosis micorriza de las comunidades asociadas a AMF varios cultivos de los agricultores cuando se cultiva en sistemas agroforestales (SAF) y en monocultivo, y estimar la diversidad de las comunidades de HMA asociadas a las raíces estas culturas. El género de HMA Glomus en la región amazónica mostró amplia adaptación a la variación ambiental en ambos sistemas, por lo que puede ser mejor estudiado en la producción de inoculantes para la plantación de árboles frutales y nativos de la Amazonía.

PALABRAS CLAVE: Amazonía, sistemas de producción, micorrizas.

\section{INTRODUÇÃO}

Os Fungos micorrízicos arbusculares (FMAs) são componentes importantes na recuperação e restabelecimento da vegetação em áreas frágeis ou degradadas, bem como na manutenção da biodiversidade de plantas e das funções dos ecossistemas (DANDAN; ZHIWEI, 2007).

Por isso, solos com baixa fertilidade como os da Amazônia, esses organismos fazem a manutenção da fertilidade dos solos, contribuindo no aumento da eficiência das plantas em absorver nutrientes, principalmente aqueles de baixa mobilidade no solo, como $\mathrm{P}, \mathrm{Zn}$ e $\mathrm{Cu}$, pois os tornam biodisponíveis (SMITH et al., 1994).

No entanto a simbiose formada pelos FMAs com as raízes das plantas pode ser afetada pelo manejo adotado no agrossistema. A colonização micorrízica pode ser elevada com adição de matéria orgânica, cultivo mínimo, sistemas de baixos insumos, diversificação de hospedeiro mediante rotação de culturas, entre outros (GALVEZ et al., 2001).
Dentro desse contexto, o objetivo desse trabalho foi avaliar a simbiose micorrízica das comunidades de FMAs associados à diversas culturas de interesse de agricultores familiares quando cultivados em sistema agroflorestal (SAF) e em monocultivo.

\section{MATERIAL E MÉTODOS}

O trabalho foi realizado e no Assentamento Agrícola Palmares no Município de Parauapebas - PA e no Assentamento Araras em São João do Araguaia - PA. As amostras de solo e raízes para identificação dos FMAs foram coletadas em áreas distintas dos Projetos de Assentamentos, em áreas de SAF's e de monocultivo.

A extração e contagem dos esporos dos FMAs do solo, foi realizada a partir de uma amostra de $50 \mathrm{~g}$ de solo, composta por 10 sub-amostras. Em seguida, foram colocados em lâminas para serem identificados de acordo com suas características morfológicas (SCHENCK; PÉREZ, 1987; INVAM, 2001). 
A identificação dos FMAs foi realizada em duas etapas, a identificação direta (ID), que é a extração dos esporos pelo método de peneiramento úmido (GERDMAN; NICHOLSON, 1963), e centrifugação em sacarose 40\% (JENKINS, 1964); e pela identificação indireta (II), onde em casa de vegetação foi colocada em vasos de 1000 g de capacidade, sementes de Brachiaria brizantha em alta densidade para forçar o desenvolvimento radicular.

Os dados foram compilados e processados através da análise de variância do teste de Tukey a uma probabilidade de $5 \%$

\section{RESULTADOS E DISCUSSÃO}

Nos sistemas de monocultivo de cupuaçu, SAFs, capoeira queimada, roça de corte e queima, roça de corte e queima em regeneração, no Projeto de Assentamento Araras foram observados a presença de seis espécies de FMAs, pertencentes ao gênero Acaulospora, Gigaspora, Glomus, Scutellospora e uma espécie com taxonomia não identificada (Tabela 1). Os gêneros que tiveram maior freqüência de ocorrência foram Glomus e Acaulospora, que ocorreram em todas as amostras avaliadas, enquanto que Gigaspora e Scutellospora apresentaram baixa freqüência de ocorrência.

No projeto de Assentamento Palmares foram caracterizadas sete espécies de FMAs na roça de mandioca e no sistema de pasto. A espécie Glomus manihots apareceu apenas no cultivo de mandioca. Os indivíduos de maior frequiência foram os gêneros Glomus, e os de menor foram Acaulospora seguida da espécie não identificada (Tabela 2).

Tabela 1. Número de esporos e frequencia (FO \%) de FMAs em $50 \mathrm{~mL}$ das amostras de solos coletadas nos sistemas de monocultivo de cupuaçu, SAFs contendo capoeira x mandioca; capoeira x babaçu; capoeira $\mathrm{x}$ castanheira; capoeira $\mathrm{x}$ mamona; mandioca $\mathrm{x}$ babaçu; leguminosa arbórea $\mathrm{x}$ mamona e sistema de capoeira queimada, sistema de roça de corte e queima e sistema de roça de corte e queima em regeneração no Projeto de Assentamento Araras, no Município de São João do Araguaia - PA (N = 10).

\begin{tabular}{lcccccc}
\hline Espécies & Cupuaçu & SAFs & $\begin{array}{c}\text { Capoeira } \\
\text { queimada }\end{array}$ & $\begin{array}{c}\text { Roça de corte } \\
\text { e queima }\end{array}$ & $\begin{array}{c}\text { Roça de corte e queima } \\
\text { em regeneração }\end{array}$ & FO (\%) \\
\hline Acaulospora escrobiculata & $12 \mathrm{Ab}$ & $22 \mathrm{Aa}$ & $0 \mathrm{Dd}$ & $0 \mathrm{Dd}$ & $3 \mathrm{Cc}$ & 4 \\
Gigaspora margarita & $0 \mathrm{Cb}$ & $8 \mathrm{Ca}$ & $0 \mathrm{Db}$ & $0 \mathrm{Db}$ & $0 \mathrm{Db}$ & 1 \\
Glomus clarum & $0 \mathrm{Cc}$ & $0 \mathrm{Dc}$ & $11 \mathrm{~B} \mathrm{~b}$ & $11 \mathrm{~B} \mathrm{~b}$ & $20 \mathrm{Aa}$ & 4 \\
Glomus etunicatum & $4 \mathrm{Bb}$ & $21 \mathrm{Aa}$ & $22 \mathrm{~A} \mathrm{a}$ & $22 \mathrm{~A} \mathrm{a}$ & $1 \mathrm{~B} \mathrm{~b}$ & $2 \mathrm{C} \mathrm{c}$ \\
Scutellospora heterogama & $4 \mathrm{Bb}$ & $12 \mathrm{Ba}$ & $1 \mathrm{C} \mathrm{c}$ & $0 \mathrm{Db}$ & $1 \mathrm{Da}$ & 2 \\
Não identificada & $0 \mathrm{Cb}$ & $0 \mathrm{Db}$ & $0 \mathrm{Db}$ & 0,1 \\
\hline
\end{tabular}

Médias seguidas da mesma letra minúscula nas linhas e maiúscula nas colunas não diferem estatisticamente entre si pelo método de Tukey (5\%) de probabilidade. 
Tabela 2. Número de esporos e frequiência (FO \%) de FMAs em $50 \mathrm{~mL}$ das amostras de solos coletadas nos sistemas de roça de mandioca e área de pastagem de braquiaria (Brachiaria brizantha) no PA Palmares, no Município de Parauapebas- PA ( $\mathrm{N}=10)$.

\begin{tabular}{lccc}
\hline \multicolumn{1}{c}{ Espécies } & Roça de mandioca & Pastagem de braquiaria (Brachiaria brizantha) & FO (\%) \\
\hline Acaulospora scrobiculata & $0 \mathrm{Db}$ & $1 \mathrm{Da}$ & 0,1 \\
Gigaspora margarita & $0 \mathrm{Db}$ & $2 \mathrm{Ca}$ & 0,2 \\
Glomus clarum & $15 \mathrm{Ca}$ & $7 \mathrm{Bb}$ & 2,2 \\
Glomus etunicatum & $20 \mathrm{Bb}$ & $49 \mathrm{Aa}$ & 6,9 \\
Glomus manihots & $23 \mathrm{Aa}$ & $0 \mathrm{Db}$ & 2,3 \\
Scutellospora heterogama & $1 \mathrm{Ad}$ & $1 \mathrm{Da}$ & 0,2 \\
Não identificada & $0 \mathrm{Db}$ & $1 \mathrm{Da}$ & 0,1 \\
\hline
\end{tabular}

Médias seguidas da mesma letra minúscula nas linhas e maiúscula nas colunas não diferem estatisticamente entre si pelo método de Tukey (5\%) de probabilidade.

A elevada freqüência do gênero Glomus no Projeto de Assentamento Palmares, confirma que o gênero possui vasta distribuição na zona tropical (SILVAJÚNIOR, 2004).

As micorrizas sofrem influência do solo e da espécie vegetal hospedeira, conseguindo altos níveis de esporulação e colonização quando o solo apresenta baixa fertilidade e condições de estresse (KLINOROMOS, 2000), como visto nos sistemas de roça de mandioca e área de pastagem de braquiaria (Brachiaria brizantha).

Os fungos encontrados já estão sendo multiplicados nas culturas-armadilhas na casa de vegetação para a posterior inoculação das mudas de espécies florestais e distribuição do inoculante.

\section{CONCLUSÕES}

Os resultados mostraram que os gêneros de FMAs Glomus na região amazônica apresentaram ampla adaptação à variação ambiental em ambos os sistemas, podendo assim, serem melhores estudados na produção de inoculantes para o plantio de mudas de espécies frutíferas e nativas da Amazônia.

\section{REFERÊNCIAS}

ALFAIA, S. S.; SILVA, N. M.; UGUEN, K.; NEVES, A. L.; DUPIN, B. Pesquisa participativa para recuperação da produtividade de sistemas agroflorestais na Amazônia ocidental: o caso do Projeto RECA, Nova Califórnia, Rondônia. 2007. Disponível em: <http://www.iamazonica.org.br/conteudo/eve ntos/biodiversidadeSolo/pdf/resumos/Painel1 _MeloE.pdf>. Acesso em: 23 mai. 2009. 
DANDAN, Z.; ZHIWEI, Z. Biodiversity of arbuscular mycorrhizal fungi in the hot-dry valley of the Jinsha River, southwest China. Appl. Soil Eco, v. 37, n. 2, p. 118-128, 2007.

GALVEZ, L.; DOUDS, D. D.; DRINKWATER, L. E.; WAGONER, P. Effect of tillage and farming system upon VAM fungus populations and mycorrhizas and nutrient uptake of maize. Plant and Soil, v.228, p. 299-308, 2001.

GERDEMANN, J. W.; NILCOLSON, T. H. Spores of mycorrhizal Endogone species extracted from soil by wt-sieving and decanting. Trans. Br. Mycol. Soc, v.46, p. 235-244, 1963.

GIOVANNETTI, M.; MOSSE, B. Na evaluation of techniques for measuring vesicular arbuscular mycorrhizal infection in roots. New Phytology. v. 84, p. 489-500, 1980.

GRACE, C.; STRIBLEY, D. P. A safer procedure for roution staining of vesiculararbuscular mycorrhizal fungi. Mycological Research. v.95, n.10, p.1160-1162, 1991.

JENKINS, W. R. A rapid centrifugal-floration technique for separating nemetodes from soil. Plant Disease Report, v.48, p. 692. 1964.

KLINOROMOS， J. N.; MACCUNE， J.; HART, M.; NEVILLE, J. The influence of arbuscular mycorrhization on the relationship between plant diversity and productivity. Ecology Letters, v. 3, n. 2, p.137-141, 2000.

KOSKE, R.E.; GEMMA, J.N. A modified procedure for roots to detect VA mycorrhizas. Mycological Research, v. 92, n. 4, p. 458488, 1989.

INTERNATIONAL

CULTURE

COLLECTION OF ARBUSCULAR \&
VESICULAR-ARBUSCULAR

MYCORRHIZAL FUNGI - INVAM, 2001.

Disponível em: <HTTP://invam.caf.wvu.edu/mycinfo/method s/cultures/monosp.htm>. Acesso em: 28 set. 2009.

NEWMAN, E .E. J. A method of estimating the total length of root sample. Journal of Applied Ecology. v.3, p.139-45, 1966.

SCHENCK, N.; PÉREZ, Y. Manual for the idenfication of VA mycorrhizal fungi. 3.ed. Gainesville: Synbergistic Publications, 1987. $286 \mathrm{p}$.

SILVA-JUNIOR, P. J. Comunidades de fungos micorrízicos arbusculares associadas à pupunha e ao cupuaçu cultivado em sistema agroflorestal em monocultivo na Amazônia Central. Piracicaba, 2004. 113 f. (Tese de Doutorado) - Escola Superior de Agricultura Luiz de Queiroz, Universidade de São Paulo.

SMITH, S. E; GIANINAZZI-PEARSON, V.; KOIDE, R.; CAIRNEY, J. W. G. Nutrient transport in mycorrhizas: structure, physiology and consequences for eficiency of the symbiosis. Plant Soil, v. 159, n. 1, p. 103113, 1994.

\section{AGRADECIMENTOS}

O presente trabalho foi realizado com o apoio da Fundação de Amparo e Pesquisa do Estado do Pará - FAPESPA e Universidade Federal do Pará - UFPA. 